\title{
L'RRK de Triomphe: a solution for LRRK2 GTPase activity?
}

Authors: Jonathon Nixon-Abell1,2, Daniel C. Berwick ${ }^{3 *}$, Kirsten Harvey ${ }^{1 *}$

\author{
Affiliations: \\ ${ }^{1}$ Department of Pharmacology, UCL School of Pharmacy, University College London, \\ London, UK \\ 2 Neurogenetics Branch, National Institute of Neurological Disorders and Stroke - \\ National Institutes of Health, Bethesda, MD, USA \\ 3 Department of Life, Health and Chemical Sciences, The Open University, Milton \\ Keynes, UK \\ *Correspondence to: \\ Kirsten Harvey \\ Department of Pharmacology \\ UCL School of Pharmacy \\ University College London \\ 29-39 Brunswick Square \\ London, WC1N 1AX, \\ United Kingdom \\ kirsten.harvey@ucl.ac.uk
}

\section{Daniel Berwick}

Department of Life, Health and Chemical Sciences

The Open University

Milton Keynes, MK7 6AA,

United Kingdom

daniel.berwick@open.ac.uk 


\title{
Keywords
}

LRRK2; Parkinson's disease; Neurodegeneration; GTPase; Protective; Wnt

\section{Abbreviations}

COR, C-terminal of Roc; GAD, GTPase activated by dimerisation; GAP, GTPase activating protein; GEF, Guanine nucleotide exchange factor; LRRK2, Leucine-rich repeat kinase 2; PD, Parkinson's disease; Roc, ras of complex proteins.

\begin{abstract}
Leucine-rich repeat kinase 2 (LRRK2) is a central protein in the pathogenesis of Parkinson's disease (PD), yet its normal function has proven stubbornly hard to elucidate. Even though it remains unclear how pathogenic mutations affect LRRK2 to cause PD, recent findings provide increasing cause for optimism. We summarise here the developing consensus over the effect of pathogenic mutations in the Roc and COR domains on LRRK2 GTPase activity. This body of work has been greatly reinforced by our own study of the protective R1398H variant contained within the LRRK2 GTPase domain. Collectively, data point toward the pathogenicity of GTP-bound LRRK2, and strengthen a working model for LRRK2 GTPase function as a GTPase-activated by dimerisation. Together with the identification of the protective R1398H variant as a valuable control for pathogenic mutations, we have no doubt that these triumphs for the LRRK2 field will accelerate research towards resolving LRRK2 function, and towards new treatments for PD.
\end{abstract}

\section{Introduction}

Parkinson's disease (PD) is the second most common neurodegenerative disorder worldwide and is characterised by resting tremor, akinesia, bradykinesia and postural instability, although additional non-motor symptoms are common. Parkinsonian symptoms coincide with the death of dopaminergic neurons in the 
substantia nigra pars compacta, with degeneration eventually spreading to other brain regions [1].

Mutations have been identified in several genes causing familial PD [2]. Of particular interest is LRRK2, which encodes Leucine-rich repeat kinase 2 (LRRK2). LRRK2 mutations account for $1-40 \%$ of PD cases in different populations, and present symptoms and brain pathologies that closely resemble idiopathic PD [2,3]. However, the precise function of LRRK2 remains a mystery.

LRRK2 consists of a catalytic core incorporating Ras of complex proteins (Roc), Cterminal of Roc (COR), and kinase domains, which is flanked by several proteinprotein interaction domains (Figure 1) [4]. Seven LRRK2 missense mutations segregate with PD, namely N1437H, and R1441C/G/H in the Roc domain, Y1699C in the COR domain, and G2019S and I2020T in the kinase domain [4]. Additional variants that increase PD susceptibility have been identified in the COR (R1628P) and WD40 (G2385R) domains [5,6].

Here we review data relating to the effect of hereditary mutations on LRRK2 GTPase function. We argue that there is an overwhelming consensus about the effect of PDcausing mutations on GTPase activity that is strengthened by our own study of the $\mathrm{R} 1398 \mathrm{H}$ protective variant. Thus in contrast to the view that the LRRK2 field is largely in disagreement, the relationship between GTPase activity and disease susceptibility may be resolved.

\section{LRRK2 GTPase Domain: GAD or small GTPase?}

The clustering of pathogenic mutations within the Roc domain, has established this domain as central to LRRK2 pathology. The Roc domain is a bona-fide GTPase [7-10], and is important for the rest of the molecule, since LRRK2 kinase activity is dependent on the binding of guanine nucleotides [11]. Nonetheless, the precise mechanism 
underpinning GTPase activity is the subject of debate, with two competing models in existence. These are summarised below.

The Ras Small GTPase Model. Based on homology to Ras-like GTPases, the LRRK2 Roc domain has been suggested to function analogously to small GTPases. Small GTPases act as molecular switches, with binding to effector proteins dictated by whether they are in the 'inactive' (GDP-bound) or 'active' (GTP-bound) states. For Ras itself, the best-described effectors are Raf kinases. These preferentially bind GTP-bound Ras, triggering Raf activation, with the hydrolysis of GTP to GDP terminating the signal [12].

Small GTPases are switched between active and inactive states by protein-protein interactions with guanine nucleotide exchange factors (GEFs) and GTPase activating proteins (GAPs). GEFs overcome the slow dissociation of GDP while GAPs enhance GTP hydrolysis (Figure 2) [13,14]. Thus, GAPs promote the GDP-bound state and are considered inhibitory, whereas GEFs promote the GTP-bound state and are considered activators. However, since GAPs and GEFs are both required for small GTPase function, these proteins have the effect of increasing GTPase activity in vitro.

Supporting this model, several publications report candidate LRRK2 GAPs and GEFs. These include ArfGAP1, which binds LRRK2 in mammalian cells and increases GTP hydrolysis in vitro [15,16], and RGS2, which is also reported to increase GTP hydrolysis [17]. The only suggested LRRK2 GEF is ARHGEF7, which enhances GTP hydrolysis and promotes GTP exchange in vitro $[18,19]$. These observations come with the caveat that LRRK2 interacts with a number of small GTPases that could be targets of ArfGAP1, RGS2 and/or ARHGEF7 and may be present in the assays as contaminants [20]. More concerningly, none of these proteins bind directly to the Roc domain. Taking these observations together, ArfGAP1, RGS2 and ARHGEF7 may modulate LRRK2 GTPase activity, but their mechanism of action is distinct from the GEFs and GAPs of small GTPases. 
The GTPase Activated by Dimerisation (GAD) Model. Advocates of the GTPase activated by dimerisation (GAD) model propose a mechanism for LRRK2 GTPase function that is based around homology with prokaryotic Roco proteins. This model differs from the small GTPase model in three key aspects (Figure 2). Firstly, the GAD model requires the LRRK2 Roc and COR domains to act as a single functional unit: a so-called RocCOR tandem domain. This idea is quite persuasive since these domains are found together throughout nature [21]. Secondly, the GAD model requires LRRK2 to function as a homodimer, with dimerisation mediated by the COR domain [22]. And thirdly, the GAD model does not require GAPs or GEFs.

Some interesting observations support this model. Most notably, a number of reports indicate that LRRK2 is largely dimeric in vivo, and dimerisation appears crucial for kinase activity [23-25]. In addition, the prokaryotic Roco structure predicts that the intramolecular Roc-COR interface is particularly important. Residues at this location are highly conserved across evolution, while a number of pathogenic LRRK2 mutations are predicted to reside at this interface. Consistently, these mutations have the same effects on GTPase activity in prokaryotic Roco proteins and human LRRK2 $[22,26]$.

\section{All PD-causing RocCOR mutations increase the proportion of GTP- bound LRRK2, whilst the protective R1398H mutation has the opposite effect}

A number of genetic linkage studies have identified an R1398H LRRK2 Roc domain mutant as a protective variant [27-29]. R1398H is the only variant of this type identified to date but has the potential to revolutionise the study of LRRK2 biology, since it can be expected to have the opposite effect to PD-causing mutations in disease-relevant processes. 
We have recently reported the effects of R1398H on LRRK2 GTPase function, and found data consistent with its status as a protective variant [30]. In particular, R1398H displayed decreased binding to GTP and increased GTP hydrolysis. Thus, R1398H appears to shift LRRK2 towards the GDP-bound state. Remarkably, this is the opposite to all reports for pathogenic RocCOR tandem domain mutations (Table 1). Taken together, these observations are consistent with GTP-bound LRRK2 being pathogenic, and raise the possibility of targeting LRRK2 GTP-binding for PD treatment. Intriguingly, a LRRK2 GTP-binding inhibitor has been reported to reduce neurodegeneration in mouse models [31]. How such compounds transfer to human PD patients remains to be seen, but their potential is promising.

We also found R1398H to increase interaction between RocCOR tandem domain constructs in yeast two-hybrid assays [30]. This is in contrast to the pathogenic R1441C/G/H and Y1699C mutations, which weaken interaction. We do not know which of the many potential inter or intramolecular interactions within a RocCOR dimer are affected, although it is unlikely to be COR-COR dimerisation, since the data are largely recapitulated in assays that use the RocCOR tandem domain as bait, but use an isolated Roc domain as prey [30]. In any case, the changes in interaction strength seen in this assay correlate with altered GTPase function: R1398H increases LRRK2 dimerisation and decreases GTP binding; pathogenic mutants have the opposite effect.

These observations are consistent with LRRK2 functioning as a dimeric GTPase that involves both Roc and COR domains, rather than as a monomeric Roc domain. Strikingly, molecular modelling of pathogenic and protective RocCOR mutations using the prokaryotic Roco structure makes this case even stronger, since the equivalent residues to R1398, N1347, R1441 and Y1699 are in remarkably close spatial proximity at the intramolecular Roc-COR interface [22,30]. In the case of R1398, mutation to histidine may prevent the formation of a Roc-COR hydrogen bond [30]. Based on this structural information, we and others have speculated that pathogenic and protective variants within the LRRK2 RocCOR domain all affect 
GTPase function by altering intramolecular Roc-COR interactions [22,30,32]. Interestingly, this has been demonstrated for the Y1699C mutation which is located within the COR domain but still affects GTPase activity, further supporting the GAD model [32]. Indeed, mutations at residues equivalent to R1441 and Y1699 in prokaryotic Roco proteins recapitulate the impaired GTP hydrolysis seen in human LRRK2 [7,22,32].

The GAD model also has serendipitous support from other studies of the R1398 site. Based on homology to Ras proteins, an artificial R1398L mutation was generated to mimic the tumorigenic Q61L substitution that renders Ras proteins constitutively GTP-bound. Contrary to expectation however, R1398L behaved like R1398H by decreasing GTP-binding and increasing GTP hydrolysis (Table 1) [15,30,33,34]. It is self-evident that behaviour opposite to that predicted from Ras is a strong argument against the small GTPase model, but the fact that R1398L would also prevent the same putative Roc-COR hydrogen bond as R1398H makes a tantalizing case for the GAD model.

\section{Pathomechanisms for mutations outside the RocCOR tandem}

\section{domain}

The above data make a compelling argument for PD-causing RocCOR mutants shifting LRRK2 to the GTP-bound state. But what about mutations outside the RocCOR tandem domain? It has long been suggested that LRRK2 kinase activity may govern GTPase function through autophosphorylation mechanisms, and in agreement with this, Roc domain autophosphorylation is reported to modulate GTPase function $[35,36]$. Based on this, one would expect mutations that alter kinase activity to indirectly affect GTPase function. However, the G2019S kinase domain mutation, which increases LRRK2 kinase activity, has been demonstrated repeatedly to have no affect on GTP-binding or GTP hydrolysis [10,15,33,34,37-39]. At the other extreme of kinase activity, an artificial kinase-dead mutation also has no effect [34]. It is perhaps surprising that the effects of altered kinase activity and autophosphorylation should 
be in conflict, but we note that autophosphorylation levels for LRRK2, even G2019S, are very low $[35,40]$. Thus, whilst the behaviour of hyper- and hypo-phosphorylated LRRK2 may be different in in vitro GTP-binding and hydrolysis assays, the range of Roc domain autophosphorylation achievable in cells may be insufficient to affect GTPase function. As such we would argue that "kinase activity controls GTPase activity" models appear unlikely (for this reason the possibility that the two enzymatic activities are connected is not included in Figure 3). The only other publication describing non-RocCOR mutations in GTPase assays is a surprising recent report of increased GTPase activity with the pathogenic I2020T kinase domain and G2385R WD40 domain mutations [41]. These observations cannot be ignored, but since they are contrary to every single publication for pathogenic RocCOR mutations, these experiments need replicating. In any case, it is clear that alternative pathomechanisms to increased GTP-binding must exist. So what could they be (Figure 3)?

The most obvious pathomechanism for non-RocCOR mutations is increased substrate phosphorylation. Substrate phosphorylation is a distinct concept to kinase activity, since the phosphorylation of substrate protein is dependent on more than just kinase activity. This is an important distinction, since G2019S remains the only mutation for which elevated kinase activity is agreed; even the adjacent I2020T mutation seems unable to elicit a reproducible effect [9,42-44] Perhaps more tellingly, the WD40 domain G2385R risk variant impairs kinase activity [44,45]. It would be surprising if both increased and decreased LRRK2 kinase activity were able to cause PD, but G2019S and G2385R could still have the same effect on substrate phosphorylation, for example by differentially affecting kinase-substrate binding. With this in mind, we note a recent report of a group of Rab GTPases (Rabs 8/10/12) as LRRK2 kinase substrates [46]. Remarkably, all pathogenic LRRK2 mutations examined increased Rab phosphorylation in cells. These included mutations that do not affect kinase activity, as well as the kinase-enhancing G2019S and kinase-impairing G2385R mutations [46]. It is worth contrasting this with LRRK2 autophosphorylation at serine-1292, which is also increased by multiple PD-causing mutations [47,48]. In 
both cases, mutations outside the kinase domain enhance substrate phosphorylation at least as well as G2019S [46-48], thereby demonstrating a lack of correlation between kinase activity and substrate phosphorylation, and illustrating the need to dissociate these concepts. Presumably non-kinase domain mutations increase serine1292 autophosphorylation by making this residue more accessible to the kinase domain. Of the two, we would argue that Rab8/10/12 phosphorylation is more likely to have pathological relevance, since the stoichiometry of in vitro Rab8/10/12 phosphorylation compares favourably to LRRK2 autophosphorylation [46]. Perhaps more importantly, serine-1292 autophosphorylation appears to be unaffected by G2385R [47]. But in any case, both are exciting stories and we await the next developments: it will be intriguing to examine how R1398H affects these phosphorylation events.

Besides its enzymatic activities, LRRK2 has a remarkable number of reported $\begin{array}{lllll}\text { interactors } & \text { (currently } & 151 & \text { on }\end{array}$ http://thebiogrid.org/125700/summary/homo-sapiens/lrrk2.html) and likely functions as a scaffold protein, nucleating multi-protein complexes through proteinprotein interactions $[49,50]$. It is self-evident that mutations in protein-protein interaction domains could alter binding to LRRK2, while RocCOR mutations have also been shown to affect binding to certain interactors [51-54]. How mutations in the kinase domain might do the same is less intuitive, although altered ATP binding and autophosphorylation are likely to affect the topology of LRRK2. In any case, there are numerous reports of altered protein binding to pathogenic LRRK2 mutants, including deregulated interactions with the G2019S and I2020T kinase domain mutants (e.g. FADD, GSK3 $\beta$, Rac1 $[52,55,56])$. It is easy to envision how changes to any one of the individual protein-protein interactions within a multi-protein complex could elicit the same functional outcome. Thus, altered protein-protein interaction is a persuasive explanation for both RocCOR and non-RocCOR mutations. 


\section{Concluding remarks}

Data from the study of pathogenic RocCOR mutations and the R1398H protective variant allow two conclusions to be drawn. Firstly, increased GTP-binding is a common pathomechanism for RocCOR mutations; and secondly, the GAD GTPase model for LRRK2 appears likely. It therefore seems reasonable to infer that GTPbound LRRK2 is a pathogenic species, and the GDP-bound form protective. This raises the possibility of treating LRRK2 PD - and perhaps idiopathic PD - with compounds that disrupt GTP binding. As mentioned, this therapeutic strategy is already under development, with encouraging results [31].

It should be reiterated that pathogenic mutations outside the RocCOR domain do not all affect LRRK2 GTPase function. Nonetheless, it is logical to assume that the effects of all pathogenic LRRK2 mutations converge at some point downstream. This point of convergence is perhaps the Holy Grail of LRRK2 biology. Currently, LRRK2 has been linked to a multitude of cellular processes, but those with pathological relevance are not agreed. However, we believe the R1398H mutant has the potential to greatly expedite LRRK2 research, since this mutant represents a powerful control that gives greater confidence to experiments producing small changes. When studied in cellular assays relevant to LRRK2 PD, R1398H can be expected to behave oppositely to PDcausing mutants. With this in mind we note that R1398H increases canonical Wnt signalling activity [30], which is in direct contrast to pathogenic variants throughout LRRK2 [30,53]. Given the established importance of properly regulated Wnt signalling for the normal function of neurons, these observations could have great implications. Of course, R1398H has yet to be studied in many of the other processes in which LRRK2 has been implicated, and may present opposite effects in some of these assays too.

A cure for LRRK2 Parkinson's disease remains a long way off, but advances are being made. In particular the data supporting a model for LRRK2 GTPase function as described herein appear overwhelming. As such, determining the role of LRRK2 
GTPase activity in PD pathogenesis may be one of the first triumphs for the LRRK2 field.

\section{Acknowledgements}

We would like to thank Simone Grannó, Vicky Spain and Craig Blackstone for their work on the R1398H project, and to acknowledge the valuable contributions of other members of the Kirsten Harvey lab, past and present, and of our collaborators in the LRRK2 field. 


\section{References}

1. Lesage, S. and Brice, A. (2009) Parkinson's disease: from monogenic forms to genetic susceptibility factors. Hum. Mol. Genet. 18, R48-59

2. Gasser, T. (2009) Molecular pathogenesis of Parkinson disease: insights from genetic studies. Expert Rev. Mol. Med. 11, e22

3. Kumari, U. and Tan, E. K. (2009) LRRK2 in Parkinson's disease: genetic and clinical studies from patients. The FEBS J. 276, 6455-6463

4. Esteves, A. R., Swerdlow, R. H. and Cardoso, S. M. (2014) LRRK2, a puzzling protein: insights into Parkinson's disease pathogenesis. Exp. Neurol. 261, 206216

5. Farrer, M. J., Stone, J. T., Lin, C. H., Dachsel, J. C., Hulihan, M. M., Haugarvoll, K., Ross, O. A. and Wu, R. M. (2007) Lrrk2 G2385R is an ancestral risk factor for Parkinson's disease in Asia. Parkinsonism Relat. Disord. 13, 89-92

6. Ross, O. A., Wu, Y. R., Lee, M. C., Funayama, M., Chen, M. L., Soto, A. I., Mata, I. F., Lee-Chen, G. J., Chen, C. M., Tang, M., Zhao, Y., Hattori, N., Farrer, M. J., Tan, E. K. and $\mathrm{Wu}$, R. M. (2008) Analysis of Lrrk2 R1628P as a risk factor for Parkinson's disease. Ann. Neurol. 64, 88-92

7. Lewis, P. A., Greggio, E., Beilina, A., Jain, S., Baker, A. and Cookson, M. R. (2007) The R1441C mutation of LRRK2 disrupts GTP hydrolysis. Biochem. Biophys. Res. Commun. 357, 668-671

8. Li, X., Tan, Y. C., Poulose, S., Olanow, C. W., Huang, X. Y. and Yue, Z. (2007) Leucine-rich repeat kinase 2 (LRRK2)/PARK8 possesses GTPase activity that is altered in familial Parkinson's disease R1441C/G mutants. J. Neurochem. 103, 238- 247

9. Liu, M., Dobson, B., Glicksman, M. A., Yue, Z. and Stein, R. L. (2010) Kinetic mechanistic studies of wild-type leucine-rich repeat kinase 2: characterization of the kinase and GTPase activities. Biochemistry. 49, 2008-2017

10. West, A. B., Moore, D. J., Choi, C., Andrabi, S. A., Li, X., Dikeman, D., Biskup, S., Zhang, Z., Lim, K. L., Dawson, V. L. and Dawson, T. M. (2007) Parkinson's disease- 
associated mutations in LRRK2 link enhanced GTP-binding and kinase activities to neuronal toxicity. Hum. Mol. Genet. 16, 223-232

11. Taymans, J. M., Vancraenenbroeck, R., Ollikainen, P., Beilina, A., Lobbestael, E., De Maeyer, M., Baekelandt, V. and Cookson, M. R. (2011) LRRK2 kinase activity is dependent on LRRK2 GTP binding capacity but independent of LRRK2 GTP binding. PloS One. 6, e23207

12. Herrmann, C., Martin, G. A. and Wittinghofer, A. (1995) Quantitative analysis of the complex between p21ras and the Ras-binding domain of the human Raf-1 protein kinase. J. Biol. Chem. 270, 2901-2905

13. Mukhopadhyay, S. and Ross, E. M. (1999) Rapid GTP binding and hydrolysis by G(q) promoted by receptor and GTPase-activating proteins. Proc. Nat. Acad. Sci. USA. 96, 9539-9544

14. Bernards, A. and Settleman, J. (2004) GAP control: regulating the regulators of small GTPases. Trends Cell Biol. 14, 377-385

15. Stafa, K., Trancikova, A., Webber, P. J., Glauser, L., West, A. B. and Moore, D. J. (2012) GTPase activity and neuronal toxicity of Parkinson's disease-associated LRRK2 is regulated by ArfGAP1. PLoS Genet. 8, e1002526

16. Xiong, Y., Yuan, C., Chen, R., Dawson, T. M. and Dawson, V. L. (2012) ArfGAP1 is a GTPase activating protein for LRRK2: reciprocal regulation of ArfGAP1 by LRRK2. J. Neurosci. 32, 3877-3886

17. Dusonchet, J., Li, H., Guillily, M., Liu, M., Stafa, K., Derada Troletti, C., Boon, J. Y., Saha, S., Glauser, L., Mamais, A., Citro, A., Youmans, K. L., Liu, L., Schneider, B. L., Aebischer, P., Yue, Z., Bandopadhyay, R., Glicksman, M. A., Moore, D. J., Collins, J. J. and Wolozin, B. (2014) A Parkinson's disease gene regulatory network identifies the signaling protein RGS2 as a modulator of LRRK2 activity and neuronal toxicity. Hum. Mol. Genet. 23, 4887-4905

18. Haebig, K., Gloeckner, C. J., Miralles, M. G., Gillardon, F., Schulte, C., Riess, O., Ueffing, M., Biskup, S. and Bonin, M. (2010) ARHGEF7 (Beta-PIX) acts as guanine nucleotide exchange factor for leucine-rich repeat kinase 2. PloS One. 5, e13762

19. Haebig, K., Gellhaar, S., Heim, B., Djuric, V., Giesert, F., Wurst, W., Walter, C., Hentrich, T., Riess, O. and Bonin, M. (2013) LRRK2 guides the actin cytoskeleton 
at growth cones together with ARHGEF7 and Tropomyosin 4. Biochim. Biophys. Acta. $1832,2352-2367$

20. Boon, J. Y., Dusonchet, J., Trengrove, C. and Wolozin, B. (2014) Interaction of LRRK2 with kinase and GTPase signaling cascades. Front. Mol. Neurosci. 7, 64

21. Bosgraaf, L. and Van Haastert, P. J. (2003) Roc, a Ras/GTPase domain in complex proteins. Biochim. Biophys. Acta. 1643, 5-10

22. Gotthardt, K., Weyand, M., Kortholt, A., Van Haastert, P. J. and Wittinghofer, A. (2008) Structure of the Roc-COR domain tandem of C. tepidum, a prokaryotic homologue of the human LRRK2 Parkinson kinase. The EMBO J. 27, 2239-2249

23. Gasper, R., Meyer, S., Gotthardt, K., Sirajuddin, M. and Wittinghofer, A. (2009) It takes two to tango: regulation of $\mathrm{G}$ proteins by dimerization. Nat. Rev. Mol. Cell Biol. 10, 423-429

24. Sen, S., Webber, P. J. and West, A. B. (2009) Dependence of leucine-rich repeat kinase 2 (LRRK2) kinase activity on dimerization. J. Biol. Chem. 284, 3634636356

25. Berger, Z., Smith, K. A. and Lavoie, M. J. (2010) Membrane localization of LRRK2 is associated with increased formation of the highly active LRRK2 dimer and changes in its phosphorylation. Biochemistry. 49, 5511-5523

26. Guo, L., Gandhi, P. N., Wang, W., Petersen, R. B., Wilson-Delfosse, A. L. and Chen, S. G. (2007) The Parkinson's disease-associated protein, leucine-rich repeat kinase 2 (LRRK2), is an authentic GTPase that stimulates kinase activity. Exp. Cell Res. $313,3658-3670$

27. Chen, L., Zhang, S., Liu, Y., Hong, H., Wang, H., Zheng, Y., Zhou, H., Chen, J., Xian, W., He, Y., Li, J., Liu, Z., Pei, Z. and Zeng, J. (2011) LRRK2 R1398H polymorphism is associated with decreased risk of Parkinson's disease in a Han Chinese population. Parkinsonism Relat. Disord. 17, 291-292

28. Ross, O. A., Soto-Ortolaza, A. I., Heckman, M. G., Aasly, J. O., Abahuni, N., Annesi, G., Bacon, J. A., Bardien, S., Bozi, M., Brice, A., Brighina, L., Van Broeckhoven, C., Carr, J., Chartier-Harlin, M. C., Dardiotis, E., Dickson, D. W., Diehl, N. N., Elbaz, A., Ferrarese, C., Ferraris, A., Fiske, B., Gibson, J. M., Gibson, R., Hadjigeorgiou, G. M., Hattori, N., Ioannidis, J. P., Jasinska-Myga, B., Jeon, B. S., Kim, Y. J., Klein, C., 
Kruger, R., Kyratzi, E., Lesage, S., Lin, C. H., Lynch, T., Maraganore, D. M., Mellick, G. D., Mutez, E., Nilsson, C., Opala, G., Park, S. S., Puschmann, A., Quattrone, A., Sharma, M., Silburn, P. A., Sohn, Y. H., Stefanis, L., Tadic, V., Theuns, J., Tomiyama, H., Uitti, R. J., Valente, E. M., van de Loo, S., Vassilatis, D. K., Vilarino-Guell, C., White, L. R., Wirdefeldt, K., Wszolek, Z. K., Wu, R. M., Farrer, M. J. and Genetic Epidemiology Of Parkinson's Disease, C. (2011) Association of LRRK2 exonic variants with susceptibility to Parkinson's disease: a case-control study. Lancet Neurol. 10, 898-908

29. Heckman, M. G., Elbaz, A., Soto-Ortolaza, A. I., Serie, D. J., Aasly, J. O., Annesi, G., Auburger, G., Bacon, J. A., Boczarska-Jedynak, M., Bozi, M., Brighina, L., ChartierHarlin, M. C., Dardiotis, E., Destee, A., Ferrarese, C., Ferraris, A., Fiske, B., Gispert, S., Hadjigeorgiou, G. M., Hattori, N., Ioannidis, J. P., Jasinska-Myga, B., Jeon, B. S., Kim, Y. J., Klein, C., Kruger, R., Kyratzi, E., Lin, C. H., Lohmann, K., Loriot, M. A., Lynch, T., Mellick, G. D., Mutez, E., Opala, G., Park, S. S., Petrucci, S., Quattrone, A., Sharma, M., Silburn, P. A., Sohn, Y. H., Stefanis, L., Tadic, V., Tomiyama, H., Uitti, R. J., Valente, E. M., Vassilatis, D. K., Vilarino-Guell, C., White, L. R., Wirdefeldt, K., Wszolek, Z. K., Wu, R. M., Xiromerisiou, G., Maraganore, D. M., Farrer, M. J., Ross, O. A. and Genetic Epidemiology Of Parkinson's Disease, C. (2014) Protective effect of LRRK2 p.R1398H on risk of Parkinson's disease is independent of MAPT and SNCA variants. Neurobiol. Aging. 35, 266 e265-214

30. Nixon-Abell, J., Berwick, D. C., Granno, S., Spain, V. A., Blackstone, C. and Harvey, K. (2016) Protective LRRK2 R1398H Variant Enhances GTPase and Wnt Signaling Activity. Front. Mol. Neurosci. 9, 18

31. Li, T., Yang, D., Zhong, S., Thomas, J. M., Xue, F., Liu, J., Kong, L., Voulalas, P., Hassan, H. E., Park, J. S., MacKerell, A. D., Jr. and Smith, W. W. (2014) Novel LRRK2 GTP-binding inhibitors reduced degeneration in Parkinson's disease cell and mouse models. Hum. Mol. Genet. 23, 6212-6222

32. Daniels, V., Vancraenenbroeck, R., Law, B. M., Greggio, E., Lobbestael, E., Gao, F., De Maeyer, M., Cookson, M. R., Harvey, K., Baekelandt, V. and Taymans, J. M. (2011) Insight into the mode of action of the LRRK2 Y1699C pathogenic mutant. J. Neurochem. 116, 304-315 
33. Xiong, Y., Coombes, C. E., Kilaru, A., Li, X., Gitler, A. D., Bowers, W. J., Dawson, V. L., Dawson, T. M. and Moore, D. J. (2010) GTPase activity plays a key role in the pathobiology of LRRK2. PLoS Genet. 6, e1000902

34. Biosa, A., Trancikova, A., Civiero, L., Glauser, L., Bubacco, L., Greggio, E. and Moore, D. J. (2013) GTPase activity regulates kinase activity and cellular phenotypes of Parkinson's disease-associated LRRK2. Hum. Mol. Genet. 22, $1140-1156$

35. Webber, P. J., Smith, A. D., Sen, S., Renfrow, M. B., and West, A. B. (2011) Autophosphorylation in the leucine-rich repeat kinase 2 (LRRK2) GTPase domain modifies kinase and GTP-binding activities. J. Mol. Biol. 412, 94-110.

36. Liu, Z., Mobley, J. A., DeLucas, L. J., Kahn, R. A., and West, A. B. (2016) LRRK2 autophosphorylation enhances its GTPase activity. FASEB J. 30, 336-347.

37. West, A. B., Moore, D. J., Biskup, S., Bugayenko, A., Smith, W. W., Ross, C. A., Dawson, V. L. and Dawson, T. M. (2005) Parkinson's disease-associated mutations in leucine-rich repeat kinase 2 augment kinase activity. Proc. Nat. Acad. Sci. USA. 102, 16842-16847

38. Pungaliya, P. P., Bai, Y., Lipinski, K., Anand, V. S., Sen, S., Brown, E. L., Bates, B., Reinhart, P. H., West, A. B., Hirst, W. D. and Braithwaite, S. P. (2010) Identification and characterization of a leucine-rich repeat kinase 2 (LRRK2) consensus phosphorylation motif. PloS One. 5, e13672

39. Aasly, J. O., Vilarino-Guell, C., Dachsel, J. C., Webber, P. J., West, A. B., Haugarvoll, K., Johansen, K. K., Toft, M., Nutt, J. G., Payami, H., Kachergus, J. M., Lincoln, S. J., Felic, A., Wider, C., Soto-Ortolaza, A. I., Cobb, S. A., White, L. R., Ross, O. A. and Farrer, M. J. (2010) Novel pathogenic LRRK2 p.Asn1437His substitution in familial Parkinson's disease. Mov. Disord. 25, 2156-2163

40. Kumar, A., Greggio, E., Beilina, A., Kaganovich, A., Chan, D., Taymans, J. M., Wolozin, B., and Cookson, M. R. (2010) The Parkinson's disease associated LRRK2 exhibits weaker in vitro phosphorylation of 4E-BP compared to autophosphorylation. PLoS One. 5, e8730. 
41. Ho, D. H., Jang, J., Joe, E. H., Son, I., Seo, H. and Seol, W. (2016) G2385R and I2020T Mutations Increase LRRK2 GTPase Activity. Biomed Res. Int. 2016, 7917128

42. Gloeckner, C. J., Kinkl, N., Schumacher, A., Braun, R. J., O'Neill, E., Meitinger, T., Kolch, W., Prokisch, H. and Ueffing, M. (2006) The Parkinson disease causing LRRK2 mutation I2020T is associated with increased kinase activity. Hum. Mol. Genet. 15, 223-232

43. Greggio, E. and Cookson, M. R. (2009) Leucine-rich repeat kinase 2 mutations and Parkinson's disease: three questions. ASN Neuro. 1

44. Jaleel, M., Nichols, R. J., Deak, M., Campbell, D. G., Gillardon, F., Knebel, A. and Alessi, D. R. (2007) LRRK2 phosphorylates moesin at threonine-558: characterization of how Parkinson's disease mutants affect kinase activity. Biochem. J. 405, 307-317

45. Rudenko, I. N., Kaganovich, A., Hauser, D. N., Beylina, A., Chia, R., Ding, J., Maric, D., Jaffe, H. and Cookson, M. R. (2012) The G2385R variant of leucine-rich repeat kinase 2 associated with Parkinson's disease is a partial loss-of-function mutation. Biochem. J. 446, 99-111

46. Steger, M., Tonelli, F., Ito, G., Davies, P., Trost, M., Vetter, M., Wachter, S., Lorentzen, E., Duddy, G., Wilson, S., Baptista, M. A., Fiske, B. K., Fell, M. J., Morrow, J. A., Reith, A. D., Alessi, D. R. and Mann, M. (2016) Phosphoproteomics reveals that Parkinson's disease kinase LRRK2 regulates a subset of Rab GTPases. eLife. 5

47. Sheng, Z., Zhang, S., Bustos, D., Kleinheinz, T., Le Pichon, C. E., Dominguez, S. L., Solanoy, H. O., Drummond, J., Zhang, X., Ding, X., Cai, F., Song, Q., Li, X., Yue, Z., van der Brug, M. P., Burdick, D. J., Gunzner-Toste, J., Chen, H., Liu, X., Estrada, A. A., Sweeney, Z. K., Scearce-Levie, K., Moffat, J. G., Kirkpatrick, D. S., and Zhu, H. (2012) Ser1292 autophosphorylation is an indicator of LRRK2 kinase activity and contributes to the cellular effects of PD mutations. Sci. Transl. Med. 4, 164ra161.

48. Reynolds, A., Doggett, E. A., Riddle, S. M., Lebakken, C. S., and Nichols, R. J. (2014) LRRK2 kinase activity and biology are not uniformly predicted by its 
autophosphorylation and cellular phosphorylation site status. Front. Mol. Neurosci. 7, 54.

49. Berwick, D. C. and Harvey, K. (2011) LRRK2 signaling pathways: the key to unlocking neurodegeneration? Trends Cell Biol. 21, 257-265

50. Lewis, P. A. and Manzoni, C. (2012) LRRK2 and human disease: a complicated question or a question of complexes? Sci. Signal. 5, pe2

51. Sancho, R. M., Law, B. M. and Harvey, K. (2009) Mutations in the LRRK2 Roc-COR tandem domain link Parkinson's disease to Wnt signalling pathways. Hum. Mol. Genet. 18, 3955-3968

52. Ho, C. C., Rideout, H. J., Ribe, E., Troy, C. M. and Dauer, W. T. (2009) The Parkinson disease protein leucine-rich repeat kinase 2 transduces death signals via Fas-associated protein with death domain and caspase-8 in a cellular model of neurodegeneration. J. Neurosci. 29, 1011-1016

53. Berwick, D. C. and Harvey, K. (2012) LRRK2 functions as a Wnt signaling scaffold, bridging cytosolic proteins and membrane-localized LRP6. Hum. Mol. Genet. 21, 4966-4979

54. Law, B. M., Spain, V. A., Leinster, V. H., Chia, R., Beilina, A., Cho, H. J., Taymans, J. M., Urban, M. K., Sancho, R. M., Blanca Ramirez, M., Biskup, S., Baekelandt, V., Cai, H., Cookson, M. R., Berwick, D. C. and Harvey, K. (2014) A direct interaction between leucine-rich repeat kinase 2 and specific beta-tubulin isoforms regulates tubulin acetylation. J. Biol. Chem. 289, 895-908

55. Lin, C. H., Tsai, P. I., Wu, R. M. and Chien, C. T. (2010) LRRK2 G2019S mutation induces dendrite degeneration through mislocalization and phosphorylation of tau by recruiting autoactivated GSK3ss. J. Neurosci. 30, 13138-13149

56. Chan, D., Citro, A., Cordy, J. M., Shen, G. C., and Wolozin, B. (2011) Rac1 protein rescues neurite retraction caused by G2019S leucine-rich repeat kinase 2 (LRRK2). J Biol Chem. 286, 16140-16149

57. Deng, J., Lewis, P. A., Greggio, E., Sluch, E., Beilina, A. and Cookson, M. R. (2008) Structure of the ROC domain from the Parkinson's disease-associated leucinerich repeat kinase 2 reveals a dimeric GTPase. Proc. Nat. Acad. Sci. USA. 105, $1499-1504$ 
58. Liao, J., Wu, C. X., Burlak, C., Zhang, S., Sahm, H., Wang, M., Zhang, Z. Y., Vogel, K. W., Federici, M., Riddle, S. M., Nichols, R. J., Liu, D., Cookson, M. R., Stone, T. A., Hoang, Q. C. (2014) Parkinson disease-associated mutation R1441H in LRRK2 prolongs the "active state" of its GTPase domain. Proc. Nat. Acad. Sci. USA. 111, 4055-4060 


\begin{tabular}{|c|c|c|c|c|c|}
\hline Mutation & $\begin{array}{l}\text { LRRK2 Construct } \\
\text { (expression in) }\end{array}$ & $\begin{array}{c}\text { GTP } \\
\text { Binding } \\
\text { (type of } \\
\text { assay) }\end{array}$ & $\begin{array}{c}\text { GTP } \\
\text { Hydrolysis } \\
\text { (type of } \\
\text { assay) }\end{array}$ & Prediction & Reference \\
\hline I1371V & Full Length (M) & $\uparrow(\mathrm{Pd})$ & ns & $\uparrow$ GTP-bound & [11] \\
\hline “I1371"V & $\begin{array}{l}\text { C.tepidum Roc + } \\
\text { COR (B) }\end{array}$ & $-(F)^{*}$ & $\downarrow \downarrow \downarrow(\mathrm{Pu})$ & $\uparrow$ GTP-bound & {$[22]$} \\
\hline “I1371"A & $\begin{array}{l}\text { C.tepidum Roc + } \\
\text { COR(B) }\end{array}$ & $-(F)^{*}$ & $\downarrow \downarrow \downarrow(\mathrm{Pu})$ & $\uparrow$ GTP-bound & {$[22]$} \\
\hline N1437H & Full Length (M) & $\uparrow \uparrow \uparrow(\mathrm{Pd})$ & ns & $\uparrow$ GTP-bound & [39] \\
\hline R1441C & Full Length (M) & $\uparrow(\mathrm{Pd})$ & $\overline{n s}$ & $\uparrow$ GTP-bound & [11] \\
\hline R1441C & Roc (M) & $-(\mathrm{R})$ & $\downarrow \downarrow(\mathrm{Pu})$ & $\uparrow$ GTP-bound & [9] \\
\hline R1441C & Full Length (M) & $-(\mathrm{Pd})$ & $\downarrow(\mathrm{Pu})$ & $\uparrow$ GTP-bound & [8] \\
\hline R1441C & Roc (B) & ns & $\downarrow(\mathrm{Pu})$ & $\uparrow$ GTP-bound & [57] \\
\hline R1441C & Full Length (M) & $\uparrow \uparrow \uparrow(\mathrm{M})$ & ns & $\uparrow$ GTP-bound & [39] \\
\hline R1441C & Full Length (M) & ns & $\downarrow$ (IP) & $\uparrow$ GTP-bound & [33] \\
\hline R1441C & $\begin{array}{c}\text { Roc + COR + kinase } \\
(\mathrm{Y})\end{array}$ & $-(\mathrm{Pd})$ & $-(\mathrm{IP})^{* *}$ & No change & [33] \\
\hline R1441C & $\begin{array}{c}\text { LRR + Roc + COR } \\
+ \text { kinase + WD40 (I) }\end{array}$ & ns & $\downarrow(\mathrm{Pu})$ & $\uparrow$ GTP-bound & {$[36]$} \\
\hline R1441C & Full Length (M) & $\uparrow \uparrow(\mathrm{Pd})$ & ns & $\uparrow$ GTP-bound & {$[16]$} \\
\hline R1441G & Full Length (M) & $\uparrow(\mathrm{Pd})$ & ns & $\uparrow$ GTP-bound & [11] \\
\hline R1441G & Roc (M) & $-(\mathrm{R})$ & $\downarrow \downarrow(\mathrm{Pu})$ & $\uparrow$ GTP-bound & [9] \\
\hline R1441G & Full Length (M) & ns & $\downarrow$ (IP) & $\uparrow$ GTP-bound & [33] \\
\hline R1441H & Roc (B) & $\uparrow(\mathrm{F})$ & $\downarrow \downarrow(\mathrm{Pu})$ & $\uparrow$ GTP-bound & {$[58]$} \\
\hline "R1441"A & $\begin{array}{l}\text { C.tepidum Roc + } \\
\text { COR (B) }\end{array}$ & $-(F)^{*}$ & $\downarrow \downarrow \downarrow(\mathrm{Pu})$ & $\uparrow$ GTP-bound & {$[22]$} \\
\hline Y1699C & Full Length (M) & $\uparrow(\mathrm{Pd})$ & ns & $\uparrow$ GTP-bound & [11] \\
\hline Y1699C & Full Length (M) & ns & $\downarrow(\mathrm{IP})$ & $\uparrow$ GTP-bound & [33] \\
\hline Y1699C & Full Length (M) & $-(\mathrm{Pd})$ & $\downarrow \downarrow(\mathrm{Pu})$ & $\uparrow$ GTP-bound & [32] \\
\hline Y1699C & Full Length (M) & $\uparrow \uparrow(\mathrm{Pd})$ & ns & $\uparrow$ GTP-bound & [16] \\
\hline "Y1699"C & $\begin{array}{l}\text { C.tepidum Roc + } \\
\text { COR (B) }\end{array}$ & $-(F)^{*}$ & $\downarrow \downarrow \downarrow(\mathrm{Pu})$ & $\uparrow$ GTP-bound & {$[22]$} \\
\hline R1398H & Full Length (M) & $\downarrow \downarrow(\mathrm{Pd})$ & $\uparrow(\mathrm{Pu})$ & $\downarrow$ GTP-bound & {$[30]$} \\
\hline T1343G & Full Length (M) & $-(\mathrm{Pd})$ & $-(\mathrm{IP})$ & - & [34] \\
\hline D1994A & Full Length (M) & $-(\mathrm{Pd})$ & $-(\mathrm{IP})$ & - & {$[34]$} \\
\hline R1398L & $\begin{array}{c}\text { Roc + COR + kinase } \\
(\mathrm{Y})\end{array}$ & $\downarrow(\mathrm{Pd})$ & $\uparrow(\mathrm{IP})$ & $\downarrow$ GTP-bound & [33] \\
\hline R1398L & Full Length (M) & ns & $\uparrow \uparrow(\mathrm{IP})$ & $\downarrow$ GTP-bound & [16] \\
\hline R1398L & Full Length (M) & $-(\mathrm{Pd})$ & $\uparrow \uparrow(\mathrm{IP})$ & $\downarrow$ GTP-bound & [34] \\
\hline R1398L/T1343V & Full Length (M) & $-(\mathrm{Pd})$ & $\downarrow \downarrow$ (IP) & $\uparrow$ GTP-bound & [34] \\
\hline R1398L/G2019S & Full Length (M) & $-(\mathrm{Pd})$ & $\uparrow \uparrow(\mathrm{IP})$ & $\downarrow$ GTP-bound & {$[34]$} \\
\hline R1398L/D1994A & Full Length (M) & $\downarrow \downarrow(\mathrm{Pd})$ & $\downarrow \downarrow$ (IP) & $?$ & [34] \\
\hline R1398L/T1343V/G2019S & Full Length (M) & $-(\mathrm{Pd})$ & $-(\mathrm{IP})$ & - & {$[34]$} \\
\hline R1398L/T1343V/D1994A & Full Length (M) & $\downarrow \downarrow(\mathrm{Pd})$ & $\downarrow \downarrow$ (IP) & $?$ & {$[34]$} \\
\hline R1398Q & Full Length (M) & $-(\mathrm{Pd})$ & $-(\mathrm{IP})$ & - & {$[34]$} \\
\hline R1398Q/T1343G & $\begin{array}{c}\text { Roc }+ \text { COR + kinase } \\
(\mathrm{Y})\end{array}$ & $\downarrow(\mathrm{Pd})$ & $\uparrow(\mathrm{IP})$ & $\downarrow$ GTP-bound & [33] \\
\hline R1398Q/T1343G & Full Length (M) & $-(\mathrm{Pd})$ & $-(\mathrm{IP})$ & - & {$[34]$} \\
\hline
\end{tabular}


Figure 1 - A schematic model of LRRK2 structure. LRRK2 contains N-terminal armadillo (ARM), ankyrin (ANK) and leucine-rich repeats (LRR), with WD40 repeats at the C-terminus. The enzymatic core is comprised of Ras-of-complex (Roc) and Cterminal of Roc (COR) domains (shown in magnified box), and a kinase domain. PDcausing mutations are shown in red, risk variants in purple, and the protective R1398H variant in green. Motifs conserved with small Ras-like GTPases are shown as white boxes and illustrate the guanine nucleotide phosphate-binding motif (P-loop - P), switch I (S1), switch II (S2) conformational switch regions, and G4 and G5 loops.

Figure 2 - Possible models of the LRRK2 GTPase. (A) A Ras small GTPase model of LRRK2 when GDP-bound (top) and GTP-bound (bottom). The action of suggested LRRK2 GEFs and GAPs is depicted. (B) A GAD-based model of LRRK2, obligate CORmediated dimeric form (top) shifting to a GTP-dependent cis-dimerisation of the Roc domains (bottom).

Figure 3 - Possible LRRK2 pathomechanisms. (A) A substrate that interacts with multiple domains of wildtype LRRK2 (black arrows), and undergoing basal levels of phosphorylation by the LRRK2 kinase domain (red arrow). (B) Substrate phosphorylation is increased directly by the G2019S mutation, or (C) indirectly by other PD-causing mutations that enhance enzyme-substrate interaction. (D) Alternatively, pathological mutations throughout LRRK2 might affect individual protein-protein interactions within a common multiprotein complex.

Table 1 - Summary of data from all publications investigating the effects of RocCOR domain mutations on LRRK2 GTPase activity. Expression systems are indicated: $\mathrm{M}=$ mammalian cells, $\mathrm{B}=$ bacteria, $\mathrm{Y}=$ yeast, $\mathrm{I}=$ insect cells. Up- and down-arrows represent statistically significant increases and decreases relative to wild-type protein. Approximate strength of change is represented by number of arrows, where one arrow indicates $\leq 1.5$-fold, two arrows between 1.5 - and 3 -fold, and three arrows greater than 3-fold; where numerical values are not reported comparisons are estimated. Types of GTP-binding experiment are represented as: Pd = pulldowns of 
LRRK2 protein from cellular lysates with GTP-coupled beads; $\mathrm{R}=$ radiolabelled nucleotide binding assays using purified protein; $\mathrm{F}$ = fluorescently labelled nucleotide binding assays using purified protein. The use of purified protein $(\mathrm{Pu})$ or immunoprecipitates (IP) in GTP hydrolysis experiments is also shown. * Indicates data referred to in text but not shown. ${ }^{* *}$ Clear but non-significant trend towards decreased GTPase activity. Note mutations at T1343 and D1994A are artificial Roc domain and kinase-dead mutations. 


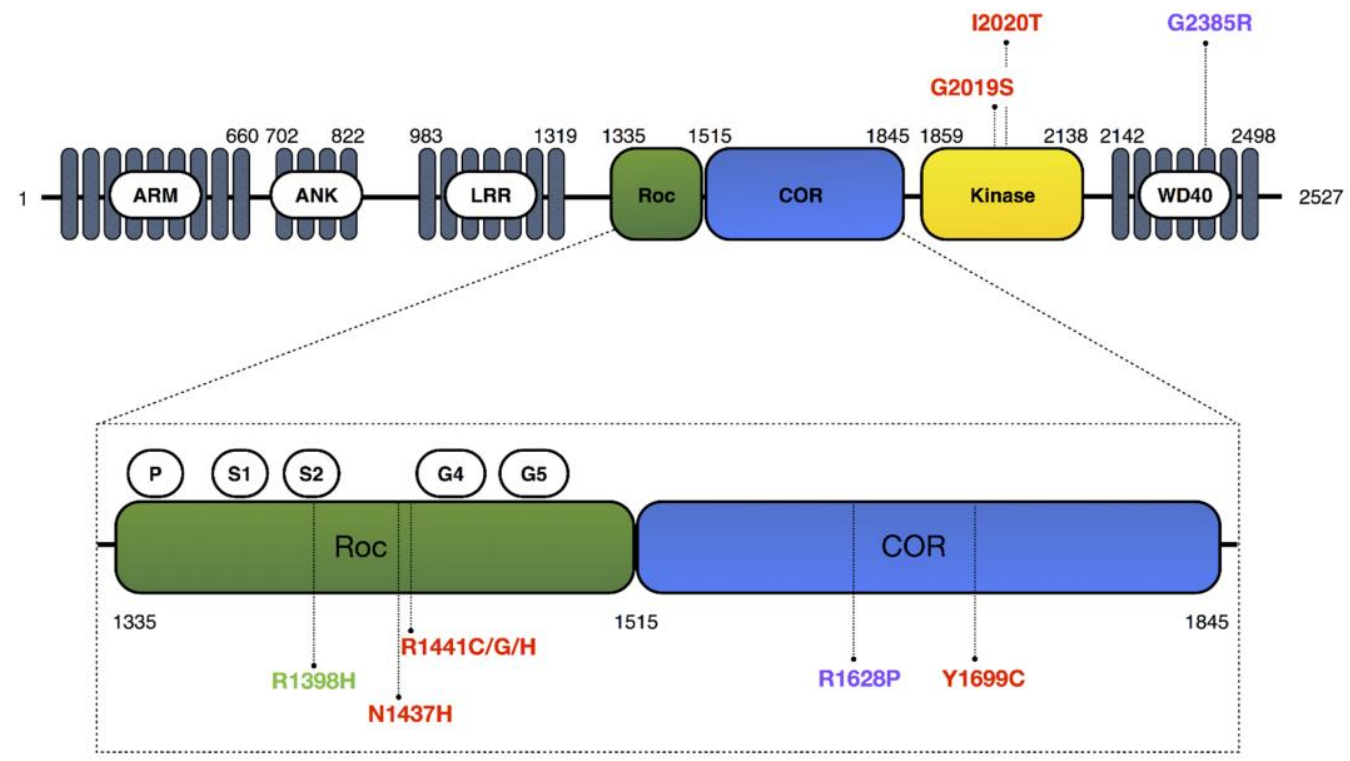


a)
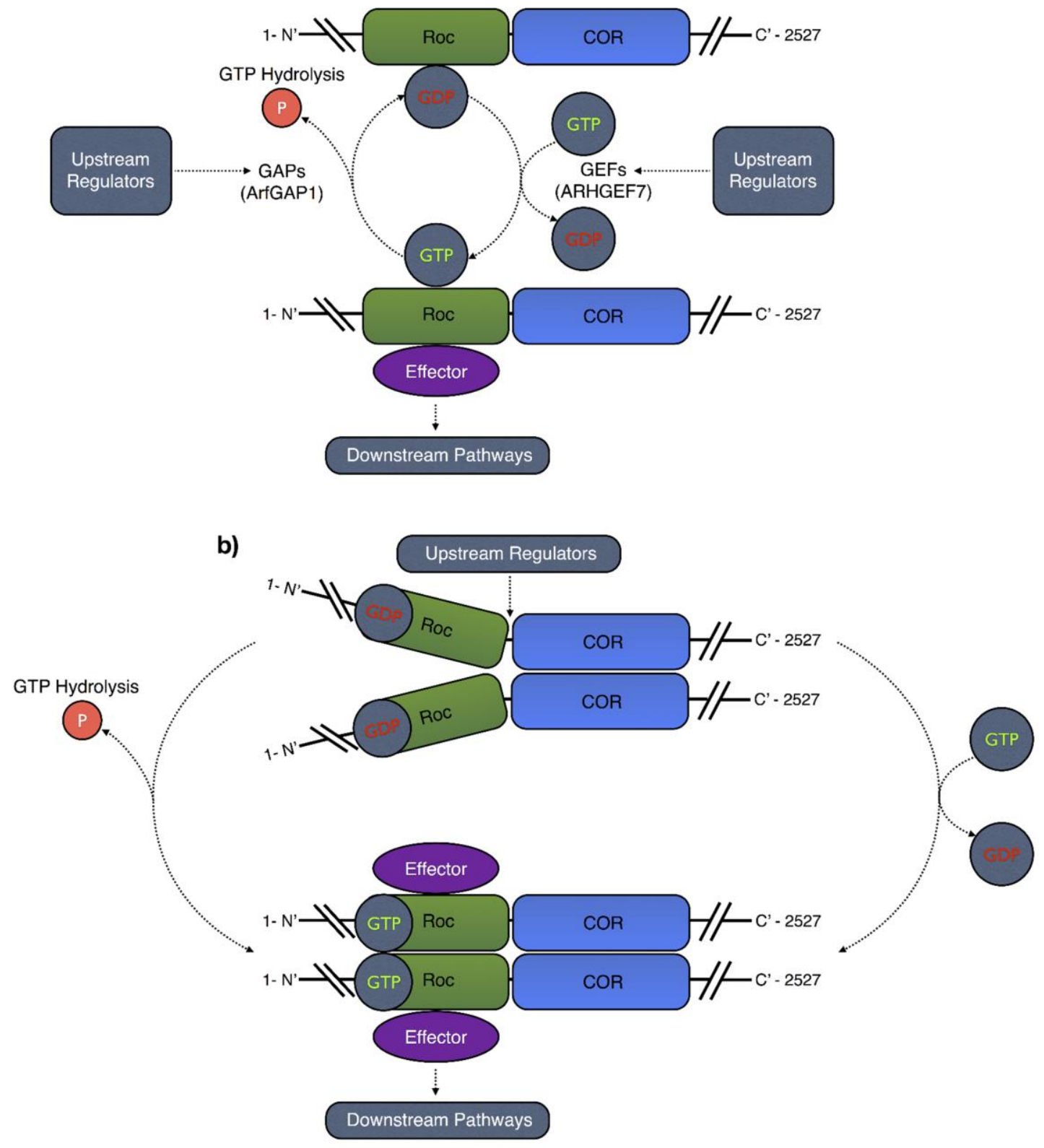

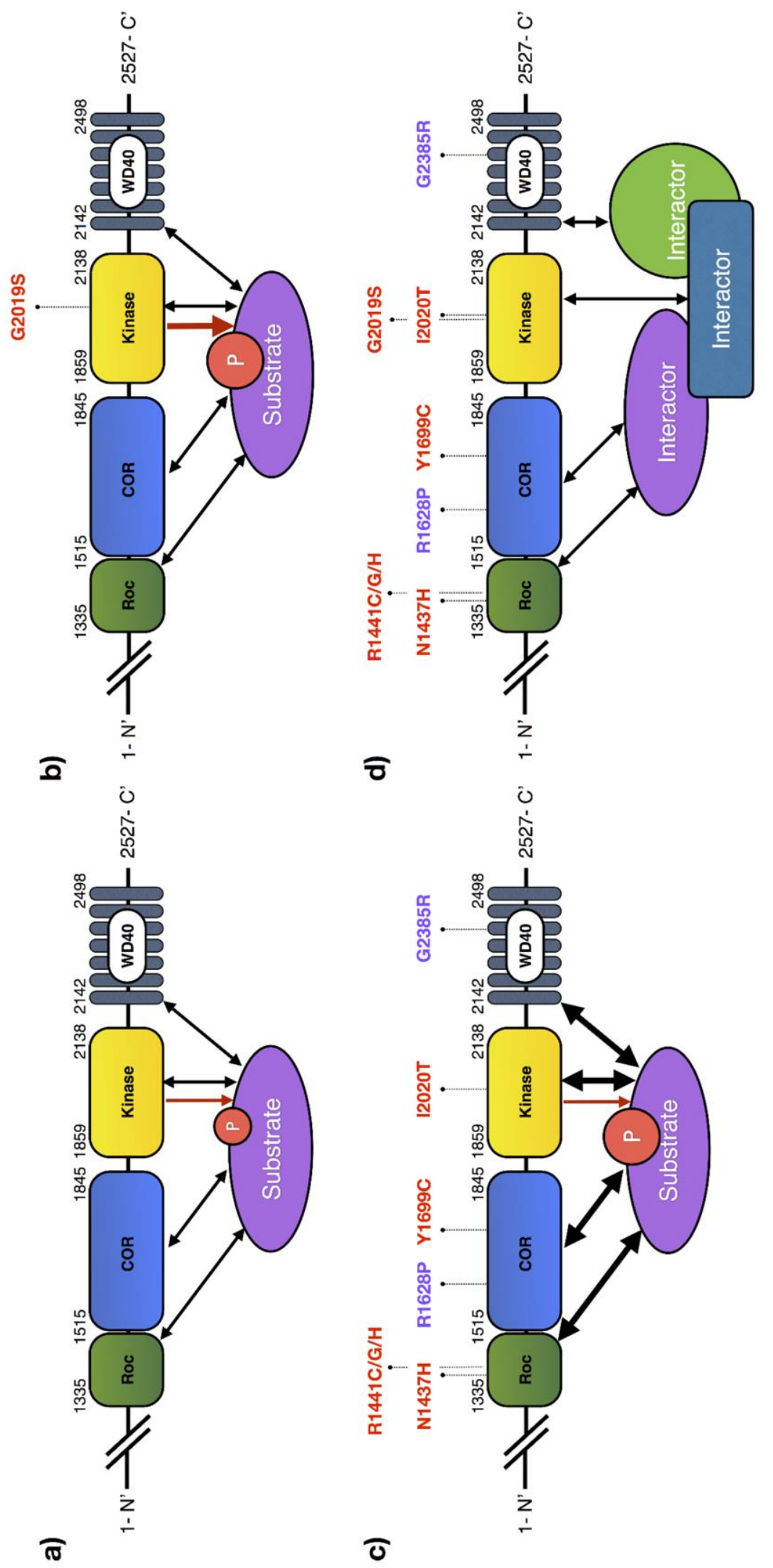